Cytogenet Genome Res 1995;69:282

\title{
Subject Index, Vol. 69, 1995
}

This index does not contain items of the reports of the Chromosome Mapping Workshops 11 and 14 nor the abstracts of the 32nd Annual American Cytogenetics Conference which are published in this volume.

American Cytogenetics Conference abstracts 110

Animals

Agalychnis callidryas (frog) 18

cattle 1,50

Chinese hamster 87

Ctenomys 179

$\operatorname{dog} 226$

goat 50

mouse $40,66,253$

Oryzomys albigularis 44

Peromyscus (deer mouse) 97

pig 33

rat $11,185,246$

sheep 50

silver fox 226

Banded chromosome analysis cattle (QFQ) 1 cattle (R) 50 Chinese hamster (G) 87 Ctenomys (C) 179 deer mouse (G) $97 \operatorname{dog}(\mathrm{G}) 226$ frog (Ag, C, G, Q) 18 goat(R) 50

human (DAPI) 15,187,223,235,273 human (G) 71,201,220,235 human(Q) 193,211,232 human (R) 38,190, 196,209 mouse (R) 66 mouse sperm (C, G) 253 Oryzomys albigularis (C, G) 44 $\operatorname{pig}(R) 33 \operatorname{rat}(Q) 185 \operatorname{sheep}(R) 50$ silver fox $(G) 226$

Chromosome aberration

$12 \mathrm{p} 27$

aneuploidy and micronuclei 215

dicentric chromosome 193

mouse Chromosome 6 in $\mathrm{Rb}$ translocations 253

rare variant of chromosome 9235

thyroid tumor cell line with $\mathrm{t}(11,19) 220$

trisomy7 90 Chromosome mapping workshops

chromosome 11 report 127

chromosome 14 report 159 Comparative gene mapping

cattle 1,50

deer mouse 97

goat 50

mouse 40, 53, 97

pig 33

rat $11,97,185,246$ 
sheep 50

Fluorescent in situ hybridization (FISH) acrocentric chromosomes and micronuclei

215 eigenanalysis of DAPI-stained chromosomes 81

G-positive band in chromosome 9 variant

235 gene mapping $1,15,33,38,50,53,75,97$,

101, 185,187,190,209,211,223,246 high-resolution R-banding 196 hncDNA library 273

microcell hybrids 63 radiation reduction hybrids 201, 240 telomeric FISH signals in immortal

Chinese

hamster cells 87 translocation breakpoint 220 trisomy 7 in nonmalignant kidney cells/

tissue 90 two-color 27

Gene mapping cattle

11 markers on chromosomes 1 to 71

BLV receptor candidate gene 50 Ctenomys

satellite DNA 179 deer mouse

Tp53, Tk1 97 goat

BLV receptor candidate gene 50 human

14cosmidson $12 \mathrm{p} 27$

15 cDNAs from human RPE library 71

68 loci on chromosome 14175

8 markers on Xp22 7

ALAS1 207

BCL1, region at llq13 101

CCNC, CCNE, CDKN1, CDKN3 190

CD38 38

chromosome 6-specific cDNA 273

GTF2F1, GTF2F2, GTF2B 75

HSD3B1.HSD3B2 59

JAK1A 232

LCN1 108

LGALS3BP 223

LIPE 211

MDK,PTN 40

MLLT2 and others on 4q1 $1 \rightarrow$ q25 260

MYH12 53

PC 187

PDE4D.PDE4B 11

SLC1A3 209

TBP 279

TPM1 15

$\mathrm{X}$-linked markers in RS region 35 mouse

Mdk, Ptn 40 rat

Pde4d, Pde4b 11

Ugtlal 185

Y-specific repeats 246 sheep

BLV receptor candidate gene 50 
Human disorders acute leukemia 260 adrenal hyperplasia 59 B-cell malignancies 101 benign thyroid tumors 220

Coffin-Lowry syndrome 7 hypophosphatemia with vitamin-D-resistant

rickets 7 juvenile retinoschisis (RS) 35 lactic acidosis 7 lymphoma 273

malignant hyperthermia susceptibility 211 malignant melanoma 273 multinodular goiters 220

Nance-Horan syndrome 7 ovarian carcinoma 273 renal cell carcinoma 90 retinoschisis 7

spondyloepiphyseal sysplasia 7

Hybrids

human-rodent 11, 40, 53, 75, 108, 207, 260 microcell hybrids 63

Radiation reduction panel 7, 201, 207, 240 rat-mouse 11

Interphase mapping

12 p 27 Interspecific backcross mapping

mouse 40

Karyotype

Agalychnis callidryas 18

Ctenomys 179

$\operatorname{dog} 226$

human, from eigenanalysis 81

mouse sperm 253

Oryzomys albigularis 44

silver fox 226

Linkage

68 loci on chromosome 14175 HSD3B1, HSD3B2 59 LIPE and markers at 19q 13211 TBP 279

X-linked RS 35

Meiosis

Agalychnis callidryas 18

human oocytes 266

male mice heterozygous for the $\mathrm{Rb}(6.16)$

translocation 253 male sterility and chromosome inversion 66

Physical mapping 4q11 $\rightarrow$ q25 260

700-kb YAC containing BCL1 101 HSD3B1, HSD3B2 59

$\mathrm{Xp} 227$

Satellite DNA

Ctenomys 179 Synaptonemal complexes

mouse, double heterozygote 66

Techniques

computer-assisted analysis of FISH 81 dual selectable markers in microcell hybrids

63 high resolution R-banding and FISH 196

282

Cytogenet Cell Genet, Vol. 69,1995 